\title{
Stimmungsbarometer Prozessleitsysteme
}

\section{Zufriedenheit von Anwendern und Lieferanten}

Qualität von Prozessleitsystemen ist eine schwer definierbare Größe. Wegen ihrer wirtschaftlichen Bedeutung und kurzer Lebenszyklen der Komponenten reicht eine Qualitätsdefinition allein auf Basis von Erfahrungswerten nicht aus. Es ist daher notwendig, dass Lieferanten nachvollziehbare Aussagen über Qualitätsmerkmale wie Lebensdauer und Ausfallwahrscheinlichkeit machen. Der Namur-AK 2.11 hat eine Umfrage durchgeführt, um die Zufriedenheit mit der aktuellen Marktsituation zu ermitteln. Aufgrund der Antworten schlägt er eine funktionsorientierte Produktsegmentierung zur Erfüllung vielfältiger Erwartungen vor.

SCHLAGWÖRTER Prozessleitsysteme / Qualitätssicherung / Namur

Mood barometer for process control systems Satisfaction of users and suppliers

It is difficult to define the quality of process control systems. Due to their economic importance and the short life cycles of the components, a definition based solely on experience is insufficient. It is thus necessary for vendors to provide information about quality features such as service life and failure probabilities of components. The Namur working group 2.11 has carried out a survey about the satisfaction with the current market situation and proposes a functionality-oriented product segmentation to fulfil a wide range of customer expectations. of Process Control Systems technology is difficult to define. Due to its high economic impact and short life cycles of the components, a definition solely based on experience is not sufficient. It is thus manatory that vendors provide information about quality features such as service life and failure probabilities of components. The Namur working group 2.11 performed a survey about the satisfaction with the current market situation and proposes a functionality-oriented product segmentation for fulfilling the existing variety of customer expectations. 


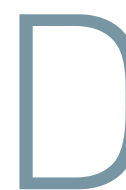

rei Jahre nach Veröffentlichung der Namur-Empfehlung NE 121 ,Qualitätssicherung leittechnischer Systeme“ [1] stellt sich die Frage, wie sich die Zufriedenheit der PLS-Anwender und Lieferanten bezüglich der in der NE 121 behandelten Qualitätsparameter darstellt. Aufgrund der Vielzahl und Komplexität dieser Parameter ist das ein schwieriges Vorhaben, das vom Namur-AK 2.11 mit der Umfrage „Zufriedenheit mit Prozessleitsystemen “ angegangen wurde. Insgesamt sind 71 Antworten eingegangen, darunter 11 Betreiber, 10 Planer, 31 Mitglieder von Fachabteilungen, hier als Experten bezeichnet, 6 Hersteller und 13 Integratoren (externe Engineeringpartner). Durch die Aufteilung in fünf Untergruppen ist die Anzahl der Antworten pro Gruppe für eine inferenzstatistische Auswertung zu gering. Trotzdem wurden die Resultate während der Vorbereitungssitzung für die Namur Hauptsitzung 2011 von allen Teilnehmern als augenscheinlich repräsentativ bezeichnet. Es ist anzumerken, dass der Arbeitskreis nicht beabsichtigt hatte, eine wissenschaftliche Untersuchung durchzuführen, sondern dass es darum ging, ein subjektives Stimmungsbild aufzuzeichnen.

\section{PRODUKTSPEZIFIKATION UND -BEURTEILUNG}

Henry Ford hat einmal gesagt: „Wenn ich die Menschen gefragt hätte, was sie wollen, hätten sie gesagt schnellere Pferde." Dies verdeutlicht die Notwendigkeit von Technologiesprüngen. Eine hohe Dynamik und Veränderung von technischen Lösungen kann noch über 100 Jahre später beobachtet werden - insbesondere bei den IT-Komponenten, welche die Grundlage der Prozessleitsysteme darstellen.

Die Erfindung des Autos führte dazu, dass die über Jahrtausende bewährte Lösung „Pferd“ plötzlich veraltet war. Damit erwiesen sich alle Erfahrungen mit der Lösung „Pferd“ als nutzlos, da sie nicht auf die neue Lösung „Auto“ übertragbar waren. Ein Beispiel: Vor der Einführung des Autos hätte ein damaliges Logistikunternehmen, zum Beispiel die Post, auf mehr Tierärzte und bessere Stethoskope gesetzt, um die bei ihr eingesetzten Transportmittel besser beurteilen zu können. Es ist allerdings frag- lich, ob diese zusätzlichen Tierärzte den Fuhrpark von Autos hätten pflegen können. Das zeigt, dass Bewertungsmethoden sich gemeinsam mit der Technologie weiterentwickeln müssen: Eine Evolution der Evaluierungsmethodik ist zwingend erforderlich.

Ein für diesen Sachverhalt repräsentatives Beispiel der jüngeren Geschichte der Leittechnik ist der technologische Fortschritt durch die Einführung von Remote I/O in Verbindung mit dem Designparameter Temperaturen, welches demonstriert, warum die Bewertung von Produkten ein nach wie vor relevantes Thema ist. In der Vergangenheit waren die I/O-Systeme in Schalträumen untergebracht, wo meist sehr technikfreundliche Umgebungstemperaturen herrschen und die zu erwartende Lebensdauer der Komponenten entsprechend hoch ist. Remote-I/O-Systeme im Feld erlauben gegebenenfalls deutliche Kosteneinsparungen, jedoch sind die Elektronikkomponenten dann eben auch rauen Umwelteinflüssen und erhöhten Temperaturen ausgesetzt: Ein schattiger Platz beschert eine längere Lebensdauer als pralle Sonne (Bild 1). Der Technologiesprung wird mit einer zusätzlichen Beurteilungsgröße erkauft, nämlich der temperaturabhängigen Lebensdauer und Ausfallrate der Systeme. Um beurteilen zu können, wie gut ein Produkt ist, muss der Anwender wissen, wie sich sein Produkt bei erhöhten Temperaturen verhält. Denn im Bereich höherer Temperaturen können Komponenten, die im Schaltraum eine durchwegs akzeptable Lebensdauer haben, sehr unterschiedlich reagieren.

Darum muss in der gemeinsamen Evolution von Technik und deren Bewertung ein neuer Designparameter, hier also die temperaturabhängige Lebensdauer, möglichst zeitnah zu einem ensprechenden neuen Bewertungsparameter auf Seite der Kunden führen.

Aktuell ist es aber meist sehr schwierig, Mean Time to Failure (MTTF) und Lebensdauerzahlen für Remote-I/OKomponenten zu erhalten, obwohl sich gezeigt hat, dass praxisrelevante Konstellationen von Produkten und Umgebungsbedingungen auftreten, die zu hohen Ausfallraten und damit Produktionsverlust führen. Diese Ausfälle bewirken dann den frühzeitigen Ersatz der I/O-Ebene.

Des Weiteren ist anzumerken, dass niedrige Ausfallraten in den ersten Betriebsjahren nicht notwendigerweise ein gu- 
ter Maßstab sind, wenn diese dann kurze Zeit später drastisch ansteigen. Als Beispiel: Fällt eine Komponente im ersten Jahr mit einer Wahrscheinlichkeit von $5 \%$ aus beträgt die MTTF 20 Jahre - aber nur, wenn das eine Jahr als typisch erklärt wird! Bei $1 \%$ Ausfallwahrscheinlichkeit wären es 100 Jahre, wenn die Komponente so lange lebt. Daraus folgt: Eine Angabe der MTTF nützt nichts, ohne die vorherbestimmte Lebensdauer einer Komponente zu kennen. Wie das Beispiel zeigt, wäre es am besten, sogar die vorgesehene Lebensdauer bei verschiedenen Betriebstemperaturen zu kennen.

Selbst die oft gescholtene IT ist hier schon weiter. Dort ist es üblich, zum Beispiel für eine Festplatte eine MTTF von 137 Jahren und gleichzeitig die vorhergesehene Lebensdauer von 5 Jahren anzugeben. Damit ist klar, dass das Laufwerk in den ersten 5 Jahren sehr zuverlässig laufen wird - 5 geteilt durch 137 führt zu 3,6 \% Ausfallwahrscheinlichkeit - dann aber eventuell zügig ersetzt werden sollte. Diese Reife der Spezifikation müssen wir auch bei Prozessleitsystemen anstreben. Bleiben die Fragen: Ist den Anwendern dieser Mangel an Information bewusst, sind sie zufrieden mit den Systemen und welche Bewertungsmethoden- und Maßstäbe werden von den Befragten eingesetzt?

\section{UMFRAGE UND PROBLEMLÖSUNGSSTRATEGIEN}

\subsection{Messbarkeit von Zufriedenheit}

Was genau bedeutet „Zufriedenheit“? Eine Definition für Zufriedenheit lautet, dass es ein Zustand ist, der aus der Erfüllung einer Erwartungshaltung resultiert. Ganz ähnlich wie „Qualität“, die sich als die Erfüllung gegebener Anforderungen definiert. Zufriedenheit ist also, je nach Fachkompetenz des Beurteilenden, direkt mit der Qualität verknüpft, wenn die Erwartungen objektiv sinnvollen Anforderungen entsprechen.

Mit welchen Methoden Qualität und Zufriedenheit gemessen werden, hängt vom jeweiligen Zielmarkt ab. Bei Konsumprodukten, zum Beispiel DVD-Spielern, wird das Qualitätsbild durch Medien, wie Computerzeitschriften oder Internetforen, geprägt. Zufriedenheit ist, wenn die Kunden wieder zurückkommen und nicht die Produkte. Die Erwartungshaltung an die Zuverlässigkeit dieser Technik ist oft sehr gering. Das steht nicht im Widerspruch zur Qualitätssicherung, zum Beispiel nach DIN ISO 9001, die explizit vom Hersteller verlangt, auch die nicht spezifizierten, aber für den bekannten Anwendungsfall notwendigen Anforderungen zu ermitteln [2].

Im Baugewerbe, als entgegengesetztes Extrembeispiel, sind die Anforderungen sehr klar durch Normen definiert. Qualität bedeutet hier Erfüllung der normativen Vorgaben. Es lässt sich vielleicht über die optischen Aspekte einer Brücke diskutieren, aber die Bauherren sind selten unterschiedlich zufrieden mit der Erfüllung der Verkehrsleistung von Brücken.

Was muss für komplexe Industrietechnik erfüllt sein, um Qualität zu gewährleisten? Eine mögliche Antwort wären die Aussagen im Lastenheft. Dieses sollte die Anforderungen möglichst genau spezifizieren, ist aber allein durch den Kunden erstellt. Der Katalog als weitere Antwortmöglichkeit hat, wie bereits erwähnt, Lücken bei Detailfragen. Erfahrungen, also eine Qualitätserfüllung durch Bewährung, scheidet aufgrund der kurzen Innovationszyklen aus. Eine rein erfahrungsbasierte Beurteilung in Kombination mit den lückenhaften Kataloginformationen ist wenig aussagekräftig und hat naturgemäß eine zu große Totzeit bezüglich der Lebensdauer. Wenn eine Komponente, von der eine Lebensdauer von 15 Jahren erwartet wird, bereits nach fünf Jahren in Massen ausfällt, dann ist es bereits zu spät. Außerdem kann die Erfahrung mit dem fünf Jahre alten Produkt aufgrund kurzer Technikzyklen nicht auf die nächsten Generationen übertragen werden.

Eine weitere Herausforderung für die Definition von $\mathrm{Zu}-$ friedenheit mit Leittechnik sind die vielen, auch teilweise widersprüchlichen Erwartungen, die von verschiedenen Branchen an ihre Produkte gestellt werden.

\subsection{Branchenabhängige Erwartungshaltung}

Die Anforderungen an Produkte sind abhängig vom jeweiligen Anwender und seiner Anwendung. Hauptfaktoren der Erwartungshaltung können folgende Punkte sein: Service und damit verbundene Aspekte wie Ersatzteilhaltung und Antrittszeiten, Funktionalität und Verfügbarkeit von Systemen, effizientes Engineering, Investions- und Lebenszykluskosten und optimierte Migrationsstrategien mit minimalen Betriebsunterbrechungen. Für eine komplexe Batchapplikation als Beispiel können die Funktionalitäten und daneben noch Service und eventuell die Migrationsabwicklung sehr wichtig sein, während die Anforderungen an die Verfügbarkeit häufig eher gering ausfallen. Für große Kontianlagen steht hingegen die Verfügbarkeit meist an erster Stelle und daneben die schnelle Abwicklung von Migrationen. Die Kosten des Leitsystems spielen bei solchen Anlagen oft eine untergeordnete Rolle. Ein für den Commodity Market produzierender Betrieb wiederum könnte die Kosten als wichtigstes Entscheidungskriterium ansehen.

Wie kann ein Lieferant mit seinen Produkten nun all diesen Anforderungen gerecht werden? Wenn er versucht, die Obermenge aller Erwartungen durch Produkteigenschaften abzudecken, liefe er in das Dilemma, höchstmögliche Zuverlässigkeit als Grundlage für eine hohe Verfügbarkeit und maximale Funktionalität bei gleichzeitigen Tiefstpreisen ermöglichen zu müssen. Das scheidet aber aus. Ziel der hier vorgestellten Strategie ist es, in einer Kooperation von Benutzern und Herstellern von Prozessleittechnik gemeinsam eine WinWin-Situation zu generieren.

\subsection{Zuverlässigkeit und Lebensdauer}

Zunächst werden die Umfrageergebnisse zum Thema Spezifikation von Zuverlässigkeit präsentiert. Wie erwähnt, finden wir in der IT-Welt für viele Produkte, darunter Festplatten, Lüfter und auch logikverarbeitende Teile wie Raid Controller, Daten bezüglich Lebensdauer und MTTF. Für elektrische Baugruppen wie Kondensatoren und Dioden gibt es diese Angaben ebenfalls.

Eine der Fragen war, ob die Lieferanten Unterlagen zu den Themen normenorientierter Entwicklung, dem Design von Elektronikbaugruppen und erwarteter Lebensdauern zur Verfügung stellen. Laut Umfrage erhalten zum Beispiel 5 von 10 Planern, die verantwortlich für die Beschaffung dieser Komponenten sind, diese Informationen und 3 weitere bekommen sie zumindest teilweise. Die 2 verbliebenen Planer stellten fest, dass solche Unterlagen kaum zur Verfügung 
gestellt werden. Es stellt sich die Frage, welche Bewertungskriterien die zufriedenen Befragten angelegt haben und ob sie eventuell mit Katalogen und den Angaben über maximal zulässige Betriebstemperaturen bereits zufrieden waren, auch wenn diese keine echten Rückschlüsse auf die Zuverlässigkeit zulassen. Wer das Fehlen notwendiger Informationen akzeptiert und eventuell MTTF und Lebensdauer verwechselt, hat Probleme, Qualität zu erkennen.

Aber möglicherweise muss uns die Zuverlässigkeit von Einzelkomponenten gar nicht interessieren, denn wir können sie ja auch redundant ausführen?

\subsection{Redundante Instrumentierung}

Das Prinzip der Redundanz ist trivial: Durch Verdoppeln der Komponenten wird die MTTF des Gesamtsystems drastisch erhöht und nur noch zufällige Doppelfehler führen zum Ausfall des Systems. Die tatsächliche technische Ausführung ist aber eventuell eine komplexe Aufgabe.

Wir fragten, ob die Betreiber und Experten durch die theoretisch längeren MTTF-Zeiten und die Möglichkeit, Reparaturen während der normalen Wochenarbeitszeit durchzuführen, einen Nutzen in Redundanz sehen.

Erwartungsgemäß bejahten 9 von 10 Betreibern diesen Nutzen. Bei den Experten sieht das Bild anders aus. Jeder dritte Experte (9 von 30) gab an, keinen Nutzen in Redundanz zu sehen. Es gab sogar Aussagen, dass Redundanz wegen der komplexeren Umsetzung zur Absenkung der Verfügbarkeit beitragen kann.

Kann man daraus schließen, dass hier detailliertes Wissen über technische Lösungen zu vermehrter Skepsis führt? Und gilt das auch in anderen technologischen Bereichen, in denen Redundanz eingesetzt wird? Ein Beispiel ist das Zweikreisbremssystem in Autos, das einen Single Point of Failure verhindert: Wenn eine der Bremsleitungen defekt ist, steht noch die Bremskraft von zwei diagonal gegenüberliegenden Bremsen zur Verfügung. Was wäre davon zu halten, wenn jeder dritte Autoexperte dieses System als zweifelhaft erachten würde?
Aber auch in Bereichen, die nicht auf Mechanik sondern Software setzen, beobachten wir eine sehr hohe Verfügbarkeit: An der Börse könnte der kleinste Systemausfall fatale Konsequenzen haben. Die Verfügbarkeit wird hier durch hochpreisige, vorkonfigurierte Serverpaare mit transparenter Redundanz sichergestellt. Warum kommen solche Systeme nicht in Leitsystemen zum Einsatz? Die Lieferanten scheinen Qualität durchaus zu kennen: In der Umfrage gaben 5 von 6 Lieferanten an, dass die „Verfügbarkeit der Systeme signifikant verbessert werden könnte, wenn eine Akzeptanz für höhere Preise auf Seiten der Kunden existierte“. Die Lieferanten sehen sich also in der Situation, aufgrund des hohen Preisdrucks am Markt in einigen Bereichen nur die zweitbeste Lösung anbieten zu können.

\subsection{Stimmungsbild Qualität}

Von allen Umfrageteilnehmern wollten wir wissen, welche Ausrichtung sie sich für die Weiterentwicklung der Leitsystemtechnik wünschen würden. Etwa die Hälfte hält die aktuelle Mischung zwischen Qualität und Funktionalität für angemessen. Dies ist der grüne Bereich des im Bild 2 gezeigten Diagramms. Wenige, darunter nur ein Betriebszugehöriger, wünschen sich einen stärkeren Fokus auf Funktionalität und der Rest plädiert für eine stärkere Konzentration auf Qualität. Es stellt sich hier also die Frage, ob die Anwender aktuell überhaupt das „schnellere Pferd“ möchten.

Bemerkenswert ist, dass ein so großer Teil der Teilnehmer sich künftig mehr Qualität wünscht. Was könnten Ursachen dafür sein? Erinnern wir uns an die Frage bezüglich der Verfügbarkeit: Sind die Kunden auch hier zu preisgetrieben, um es dem Hersteller zu ermöglichen, Qualität zu liefern? Alle Hersteller stimmen dieser These in der Umfrage zu! Aber auch 7 der 11 Betreiber geben zu, dass der Preisdruck zu hoch ist. Bei den Planern, die in letzter Konsequenz die Systeme bestellen, halten nur 2 der 10 Teilnehmenden den Preisdruck für zu hoch.

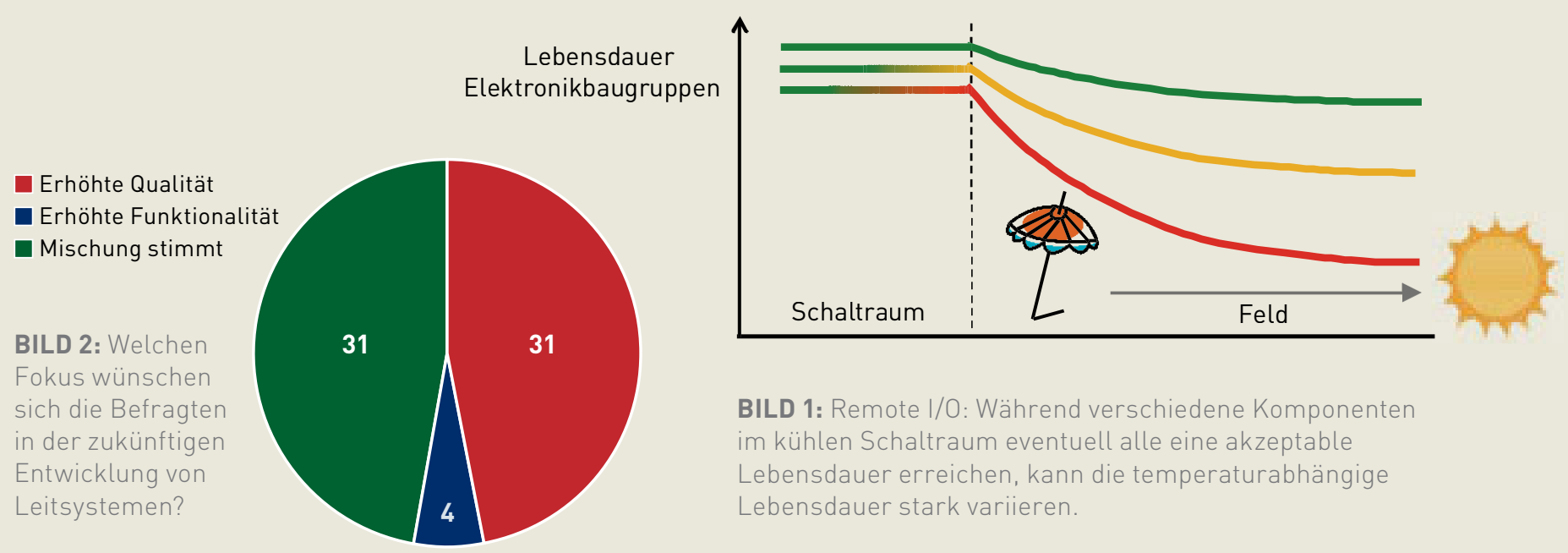




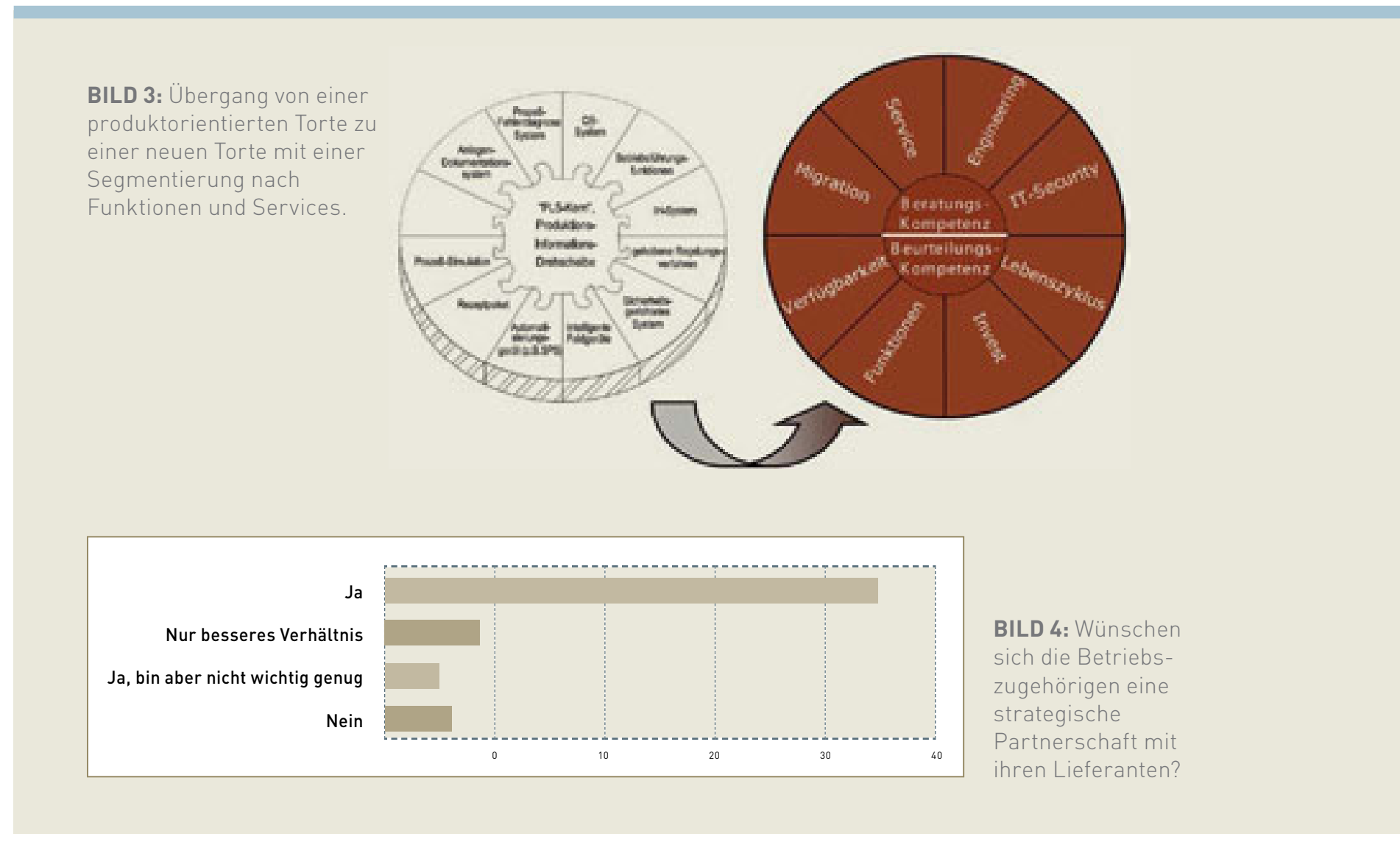

\subsection{Modulare Systemgestaltung}

Wie können Kunden mit ihren unterschiedlichen Anwendungen und Erwartungen nun eine für sie optimale Lösung zum angemessenen Preis erhalten? Ein modularisierter Ansatz ist die Tauchnitz'sche Torte [3], die auf der linken Seite von Bild 3 gezeigt ist. Die Idee dieser mittlerweile 15 Jahre alten Torte ist es, Produkte unterschiedlicher Hersteller wie Batch und intelligente Feldgeräte mit Hilfe eines zentralen Leitsystemkerns zu verbinden. Dieser Ansatz ermöglicht es, Aspekte, auf die ein Anwender besonderen Wert legt, mit entsprechend hochqualitalitativen Produkten, „Premiumstücken“, abzudecken, sodass eine kundenspezifische Kostenstruktur entsteht. Das Problem, das eine Umsetzung der Torte verhindert, ist die Inkompatibilität von Produkten verschiedener Hersteller oder gar die Inkompatibilität verschiedener Produktreihen eines Herstellers.

Wir stellen in diesem Beitrag einen neuen Ansatz vor, der den modularen Gedanken des Tauchnitz'schen Kuchens aufnimmt aber die Segmente nicht nach Produkten und Komponenten sondern nach Service- und Funktionalitätsaspekten unterteilt. Tortenstücke sind für uns die zuvor genannten Hauptfaktoren wie Verfügbarkeit, Service und Invest, die nicht unbedingt einzelnen Produkten zugeordnet werden können.

Der Kern der Torte ist nicht länger technologischer Natur. Er besteht nun aus der Beratungskompetenz der Lieferanten und der Beurteilungskompetenz der Betriebszugehörigen. Eine Kompetenz in Beratung und Beurteilung vorausgesetzt, kann der Lieferant dem Kunden helfen, die für ihn richtige Torte zu fertigen, das heißt die einzelnen Faktoren so auf seine Bedürfnisse anzupassen, dass alle Erwartungen erfüllt werden und gleichzeitig ein bestmöglicher Preis erzielt wird. Auch wenn ein ausreichend breites Portfolio an kompatiblen Produkten Voraussetzung für diesen Ansatz ist, geht es dabei nicht primär um Produkteigenschaften sondern um Beratung. Wenn ein Anwender hohe Anforderungen an Remote-I/O-Komponenten stellt, kann dies neben besserer Technik auch durch Beratung zum Thermomanagement (Sonnenschutz, Ventilation) erreicht werden.

Diese Form der Beratung setzt ein enges Verhältnis zwischen Kunden und Herstellern voraus, da der Hersteller die Anforderungen seiner Kunden sehr genau kennen und verstehen muss. Sind die Kunden dafür bereit? Wir fragten: „Wünschen Sie sich eine strategische Partnerschaft mit ihrem Lieferanten?" Die überwältigende Mehrheit ist interessiert. 36 von 53 Betriebszugehörigen antworten mit ,ja“, 7 wünschen sich zumindest ein besseres Verhältnis, 4 halten sich nicht für wichtig genug und nur 5 antworten mit „nein“. Die Vorzeichen für eine erfolgreiche Kooperation stehen also gut.

\section{ZUSAMMENFASSUNG UND AUSBLICK}

Das Erkennen von Qualität setzt Beurteilungskompetenz beim Kunden voraus. In der zeitlichen Entwicklung bedeutet dies, dass eine gemeinsame Evolution von Technik und Bewertungsmethodik stattfinden muss. Zur Erfüllung vielfältiger Kundenerwartungen bei gleichzeitig akzeptablen Preisen schlagen wir eine funktionale Segmentierung des Produktportfolios vor, von dem die richtigen Tortenstücke durch kundennahe Beratung ausgewählt werden. 
Wichtig für diesen Ansatz ist, dass die Definition von Premiumstücken vorhanden sein muss und sie auch nachgefragt wird. Auf dem europäischen Markt ist die Nachfrage schwach. Die Lieferanten können deshalb einige Services fast nicht mehr anbieten. Dies führt dazu, dass Komponenten in Saudi Arabien teilweise eine längere Lebensdauer haben als bei uns. Das unterstreicht noch einmal die Wichtigkeit von kompetenter Beratung bei Aspekten wie dem Thermomanagement.

Den Herstellern muss die Chance gegeben werden, den Anwendern Konzepte für den optimalen Einsatz ihrer Komponenten vorzustellen. Wenn die Betreiber die Hersteller als reine Teilelieferanten betrachten ist das langfristig schädlich. Um die Produktion in Europa nachhaltig wirtschaftlich zu gestalten, ist technische Exzellenz unerlässlich. Dies liegt im Interesse von Betreibern und Lieferanten.

Bei der Planung von Leitsystemen können sich die Entscheider unter dem Zeitdruck einer Projektvergabe nicht lange mit technischen Details aufhalten. Wenn die Lieferanten sich nicht durch ausreichende Spezifikation ihrer Lösungen unterscheidbar machen, ist der Preis das einzig mögliche Bewertungskriterium. Das würde sich ändern, wenn ein möglichst großer Bereich der Torte bereits im Projektvorfeld geklärt wäre. Dies kann durch Konzepte innerhalb von strategischen Partnerschaften gelingen, bei denen im Projektvorfeld qualifizierte Lösungen und Pakete zum Einsatz kommen.

Ein „Premium PLS“ im Sinne dieses Beitrags ist ein Leitsystem, dass die individuellen Erwartungen kostenoptimal aber vollständig erfüllt, entweder durch Produkteigenschaften oder Applikation. Wir möchten es nicht einfach bei der Anregung zur besseren Zusammenarbeit belassen sondern die weitere Konkretisierung und Ausarbeitung von klaren und praxisgerechten Qualitätskenngrößen in der Form von Arbeitskreisen initiieren. In diesen Gremien möchte der Arbeitskreis 2.11 „Industrielle Leittechnik“ zusammen mit den Lieferanten und weiteren Teilnehmern die Definition von Premiumstücken vornehmen. Ausgangspunkt für die Zusammenarbeit soll das Beispiel der Zuverlässigkeit in Verbindung mit der temperaturabhängigen Lebensdauer von Elektronikkomponenten bilden. Ein Anschlussthema könnte beispielsweise die anforderungsgerechte Auswahl und Gestaltung von Feldbussen sein.

Ziel dieser Kooperation sind dokumentierte Problemlösungen, welche die Beschaffer von Leitsystemen als qualitätsgesicherte Premiumstücke von den angefragten Lieferanten leicht erhalten können.

\section{REFERENZEN}

[1] Namur Empfehlung NE 121 „Qualitätssicherung leittechnischer Systeme", Namur Geschäftsstelle, www.namur.de, 2008

[2] Tauchnitz, T.: Die „neuen Prozessleitsysteme“ - Wohin geht die Reise?. atp - Automatisierungstechnische Praxis 38 (1996) H. 11, S. 12-23

[3] Deutsches Institut für Normung: DIN EN ISO 9001: 2008, Beuth-Verlag, 2008

\section{AUTOREN}

Dipl.-Ing. MARTIN BRAND (geb. 1961) ist seit 1984 bei der Evonik im Bereich Engineering/ Automatisierungstechnik tätig. Themenenschwerpunkte sind Betriebsdatenerfassungsund MES Systeme.

Evonik Industries AG,

TE-EN-E, D-63457 Hanau-Wolfgang,

Tel. +49 (0) 6181594733 ,

E-Mail: martin.branddevonik.com

Dr.-Ing. ULRICH CHRISTMANN (geb. 1966) arbeitet seit 1999 in verschiedenen Bereichen der Prozessautomatisierung für Bayer.

Bayer Technology Services $\mathrm{GmbH}$, D-51386 Leverkusen,

Tel. +49 (0) 2143074239 ,

E-Mail: ulrich.christmannabayer.com

Dipl.-Ing. MICHAEL DIEFENBACH (geb. 1959) war seit 1982 in verschiedenen Firmen auf dem Gebiet der Automatisierungstechnik beschäftigt und ist seit 2009 bei der Akzo Nobel Industrial Chemicals in den Technischen Diensten im Arbeitsgebiet PLT-Projekte und Betriebsbetreuung tätig.

Akzo Nobel Industrial Chemicals, D-65926 Frankfurt am Main,

Tel. +49 (0) 693057697 ,

E-Mail: michael.diefenbachđakzonobel.com

Dr.-Ing. THOMAS HAUFF (geb. 1960) ist im Fachzentrum Automatisierungstechnik auf dem Arbeitsgebiet der Prozessleittechnik tätig. Themengebiete sind unter anderem Qualitätssicherung, technische Evaluierung und Consulting für Automatisierungslösungen. Er ist Obmann des Namur AK 2.11.

BASF SE,

D-67056 Ludwigshafen, Tel. +49 (0) 62160203 26,

E-Mail: thomas.hauffabasf.com

Dr. rer. nat. MICHAEL KRAUß (geb. 1984) ist seit 2010 bei der BASF SE, Ludwigshafen, im Fachzentrum Automatisierungstechnik auf dem Gebiet der Prozessleittechnik tätig. Schwerpunkte sind Projekte auf dem Gebiet der Leitsystemmigrationen, Höherautomatisierung und modellbasierter Anwendungen.

BASF SE,

D-67056 Ludwigshafen, Tel. +49 (0) 6216046694 , E-Mail: michael.krauss@abasf.com 